Ann. Zootech., I975, 24 (4), 65I-660.

\title{
ÉTUDE DE DEUX TECHNIQUES DE DIAGNOSTIC DE GESTATION CHEZ LA TRUIE
}

\author{
M. J. BOSC, Françoise MARTINAT-BOTTE* et Agnès NICOLLE \\ Station de Physiologie de la Reproduction, \\ Centre de Recherches de Tours, I. N.R. A., \\ Nouzilly, 37380 Monnaie \\ * I. T. P., 149, rue de Bercy, \\ 75579 Paris Cedex 12
}

RÉSUMÉ

Deux techniques de diagnostic de gestation ont été étudiées chez la Truie.

La première est basée sur l'observation d'un retour éventuel en ostrus 18 à 25 jours après l'insémination de référence. L'exactitude totale varie suivant les élevages de 73 à 93 p. Ioo, elle est égale à 100,0 p. Ioo pour les truies gravides, elle est très faible pour les truies non gravides. Sur ces dernières, l'exactitude n'est pas améliorée lorsqu'on administre iq jours après insémination un mélange œstrogène-androgène. Il en est de même lorsque ce traitement est réalisé 25 jours après saillie et que l'œestrus est détecté du $25^{\mathrm{e}}$ au $3 \mathrm{I}^{\mathrm{e}}$ jour.

La deuxième technique est celle de la biopsie vaginale. L'exactitude totale est de l'ordre de 92 p. Ioo pour les truies gravides elle est de 96,7 p. I oo et de 71,5 p. Ioo pour les non-gravides. Par cette technique l'état de non-gravidité peut être décelé sur 68 p. I oo des animaux qui présentent des retours en cestrus tardifs.

\section{INTRODUCTION}

Chez la Truie, la non-réapparition du comportement d'œstrus après l'insémination artificielle permet habituellement de présumer de l'état de gravidité. Or, les conditions de l'élevage moderne, 1'augmentation des effectifs, la rationalisation du travail réduisent le temps de contact de l'éleveur avec ses animaux et rendent plus difficile une détection précise des chaleurs. Cette évolution de l'élevage, l'importance économique des erreurs (CAUGANT, I974) ont conduit à rechercher des techniques de diagnostic de gestation sûres et s'intégrant sans difficultés au programme de travail de 1'élevage. Pour cela, certains ont cherché à induire et à grouper ce comportement en utilisant les propriétés des cestrogènes. En effet, chez la Truie castrée, en l'absence de progestérone, l'administration de benzoate d'œstradiol ou de propionate de testos- 
térone provoque les chaleurs après un délai de 70 heures environ (SIGNORET, r967). Chez la Truie non gravide, la situation hormonale est identique car après la chute du taux de progestérone (STABENFELDT et al. I I969) et avant l'ovulation, on constate une augmentation brutale du taux des œestrogènes (SHEARER et al., I972 ; ROBERTSON et KING, I974). On conçoit alors l'intérêt d'un traitement œstrogénique au moment du retour éventuel en œstrus pour différencier les truies non gravides des truies gravides qui ont à ce moment une progestéronémie élevée (RoBERTson et SARDA, I97I). Certaines techniques basées sur cette propriété des œestrogènes ont déjà été proposées aux éleveurs (JöchlE et ScHILIING, I965; JöchlE et al., I967 ; Rothe, I970 ; ANDRÄ et al., I969; MAIRE et al., I973 ; BECzE et SzILvassy, I97I) ; on observe alors les chaleurs sur les animaux non gravides bien qu'on puisse penser qu'elles se soient produites normalement. Pour éprouver l'efficacité d'un tel traitement hormonal, nous avons repris cette étude dont nous présentons ici les résultats.

A 1'opposé de cette technique qui nécessite une surveillance assez longue des animaux, des moyens de diagnostic de gestation plus systématiques ont été développés. C'est le cas de la biopsie vaginale dont le principe résulte des différences de morphologie de l'épithélium vaginal entre l'état de gestation et de celui de la non-gestation. Les taux d'exactitude qui ont été rapportés (WALKER, I972 ; SMIDT, I973 ; RoMmEL et al., I973; GIRARDOT, I97I) sont élevés. Précédemment, nous avions indiqué que cette technique permettrait peut-être de déceler les truies présentant des retours en chaleurs tardifs (GrRARDoT et al., I972). Nous rapportons ici les observations réalisées sur un grand nombre d'animaux pour infirmer ou confirmer ces résultats.

\section{MATÉRIEL ET MÉTHODES}

a) La première expérience s'est déroulée dans un élevage expérimental ne comportant que des truies nullipares de race Large White. La technique de diagnostic de gestation est l'observation d'un retour éventuel en chaleurs I 8 à 25 jours après l'insémination de référence.

Les truies ont été divisées en deux lots : un lot de témoins sans aucun traitement, un lot de traitées. Ces dernières ont reçu chacune le $19 \mathrm{e}$ jour après l'insémination une injection $(2 \mathrm{ml})$ de $2 \mathrm{mg}$ de valérianate d'œstradiol et de $5 \mathrm{mg}$ d'œnanthate de testostérone (mélange hormonal souvent utilisé pour induire le comportement d'ustrus).

Pour chaque truie, deux modes de détection de l'œstrus ont été comparés, le premier par le réflexe d'immobilisation (par chevauchement), le deuxième par le verrat présenté quotidiennement aux femelles pendant la période d'observation. Ces deux modes de détection ont été effectués indépendamment l'un de l'autre bien que les stimuli d'ordre olfactif n'aient pas pu être contrôlés avec rigueur (Signoret et du Mesnil du Buisson, I96I).

b) La deuxième expérience s'est déroulée dans 8 troupeaux différents (races Large White, Landrace, Hybrides Large White, Landrace ou Camborough).

La technique de diagnostic utilisée est le comportement d'œestrus observé suivant la façon habituelle de chaque élevage (en général sans le verrat) après administration d'un produit témoin ou d'un produit destiné à provoquer les chaleurs.

Deux produits ont été étudiés : le produit I contient I $\mathrm{mg}$ de valérate d'œstradiol et $2,5 \mathrm{mg}$ de propionate de testostérone par $\mathrm{ml}$ de solution huileuse ; le produit II est un mélange de $\mathrm{I} \mathrm{mg}$ de valérianate d'cestradiol et de $2,5 \mathrm{mg}$ d'onanthate de testostérone par $\mathrm{ml}$ également. Le volume administré a été de $2 \mathrm{ml}$ par truie quel que soit le produit. Un seul produit a été utilisé par élevage ; il a été chaque fois comparé au solvant ou produit témoin $(2 \mathrm{ml}$ d'huile d'olive ou de germe de maïs). Afin de rendre équitable la comparaison entre le produit témoin et les produits I et II, ceux-ci ont été présentés sous le mème conditionnement et avec un nom de code variant d'un élevage à l'autre. La répartition des animaux a été faite au hasard.

Deux moments d'administration ont été étudiés (un seul par élevage), le $19^{\mathbf{e}}$ ou le $25^{\mathbf{e}}$ jour après l'insémination. Dans chaque troupeau, toutes les truies inséminées sont soumises au diagnostic de gestation. 
Le diagnostic a été exact lorsque les truies gravides ne sont pas revenues en œestrus après le traitement et lorsque les truies non gravides ont présenté des chaleurs au cours des 7 jours qui ont suivi l'injection ( $\mathrm{du} \mathrm{I}^{\mathrm{e}}$ au $25^{\mathrm{e}}$ jour après insémination pour le traitement à $\mathrm{r} 9$ jours ; du $25^{\mathrm{e}}$ au $3 \mathrm{I}^{\mathbf{e}}$ jour pour le traitement au $25^{\mathrm{e}}$ jour). Dans le cas contraire, le diagnostic a été considéré comme inexact.

c) La troisième expérience s'est déroulée dans 4 élevages sur des truies de races diverses (Large White, Piétrain, Hampshire, Landrace et croisées Large White-Landrace).

Le diagnostic de gestation est porté après examen de l'épaisseur et de l'aspect qualitatif de l'épithélium vaginal ainsi que nous l'avons précédemment défini (GIRARdot et al., I972).

Le prélèvement de la biopsie est effectué par l'éleveur avec une pince adéquate dans l'aire ventrale antérieure du vagin entre le $\mathbf{I}^{\mathrm{e}}$ et le $29^{\mathrm{e}}$ jour après la saillie. La pièce de muqueuse vaginale ainsi extraite est immédiatement fixée dans le mélange Bouin Hollande acétique et envoyée au laboratoire où elle est préparée pour l'observation microscopique suivant une technique histologique classique.

d) A la fin de chaque expérience, les diagnostics de gestation ont été comparés à l'état de gravidité jugé par la mise bas, l'avortement ou l'abattage, et à l'état de non gravidité jugé par les retours en chaleurs. Les animaux qui ont été réformés sans aucun contrôle de l'état de gravidité ou de non-gravidité ont été éliminés. De plus, les truies réformées entre I Io et I 50 jours après l'insémination sans qu'il n'y ait eu mise bas ont été considérées comme non gravides.

Pour l'analyse, l'exactitude des diagnostics est définie en pourcentage par le rapport du nombre des diagnostics exacts sur le nombre total de diagnostics effectués. Cette exactitude a été calculée, par élevage, ou par technique, ou par état physiologique.

e) Enfin, au cours de la deuxième expérience, le taux de fertilité des truies inséminées au moment du retour en œstrus a été calculé.

\section{RÉSULTATS}

Au cours des deux premières expériences, l'exactitude du diagnostic sur les truies gravides a été pratiquement égale à roo,o p. Ioo (aucun retour en cestrus n'a été constaté excepté une truie de l'élevage E), quelles que soient les conditions expérimentales.

\section{TABLEAU I}

Diagnostic de gestation :

comparaison de deux modes de détection de l'xestrus (induit ou non) chez la Truie

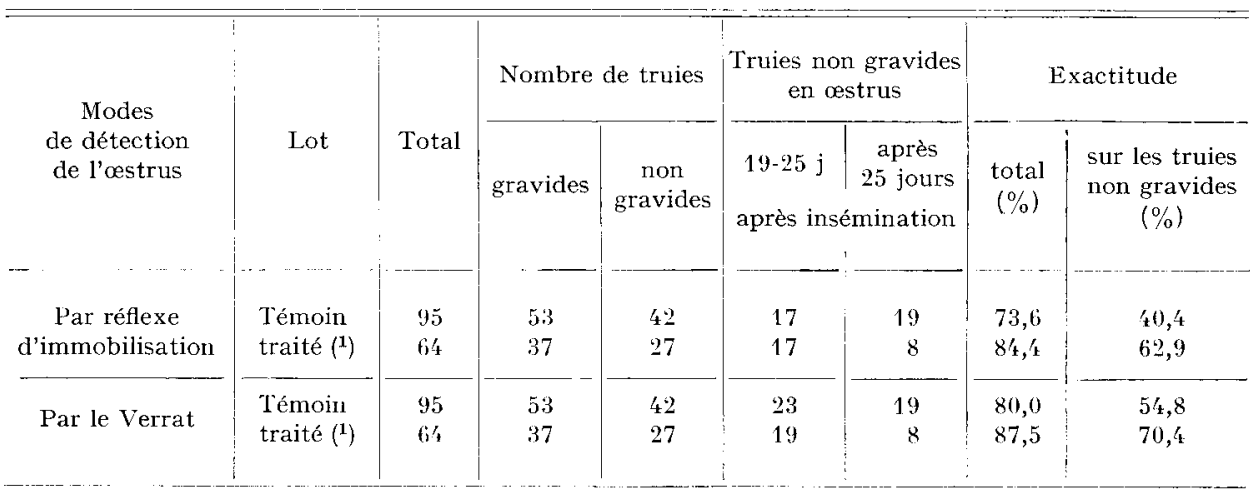

(1) Chaque Truie a reçu 19 jours après insémination $2 \mathrm{mg}$ de valérianate d'œstradiol et $5 \mathrm{mg}$ d'œnanthate de testostérone.

Exactitude : (nombre de diagnostics exacts/nombre de diagnostics) $\times 100$.

N. $B$. : L'exactitude sur les truies gravides est de 100 p. 100 dans chaque lot. 
Les résultats de la première expérience sont présentés dans le tableau I. Lorsque l'œestrus est détecté par le verrat, l'exactitude totale est égale à 80,0 p. Ioo; elle n'est que de 54,8 p. roo pour les animaux non gravides. Après traitement par le valérianate d'ostradiol et l'wenanthate de testostérone 19 jours après insémination, ces exactitudes augmentent de façon non significative ; elles atteignent respectivement 87,5 p. Ioo et $70,+\mathrm{p}$. Ioo $(\mathrm{P}<0,05)$. Lorsqu'on ne tient pas compte du réflexe d'immobilisation, l'exactitude totale et celle obtenue sur les animaux non gravides ne diffèrent pas, mais elles sont cependant plus faibles $(P<0,05)$; elles sont respectivement égales à $73,6 \mathrm{p}$. I oo et $40,4 \mathrm{p}$. Ioo pour les témoins et à $84,4 \mathrm{p}$. I 00 et 62,9 p. I 00 pour les traités.

Les résultats de la deuxième expérience où ont été comparés un mélange hormonal (produit I ou II) au produit témoin par administration 19 jours après insémination figurent dans le tableau 2.

\section{TABLEAU 2}

Diagnostic de gestation par induction du comportement d'cestrus 19 jours après insémination chez la Truie.

\begin{tabular}{|c|c|c|c|c|c|c|c|}
\hline \multirow[t]{2}{*}{ Élevage } & \multirow[t]{2}{*}{ Produit } & \multicolumn{2}{|c|}{ Nombre de truies } & \multicolumn{2}{|c|}{$\begin{array}{l}\text { Truies non gravides } \\
\text { cestrus } \\
\text { après insémination }\end{array}$} & \multirow{2}{*}{$\begin{array}{c}\text { Exactitude } \\
\text { totale } \\
(\%)\end{array}$} & \multirow{2}{*}{$\begin{array}{c}\text { Exactitude } \\
\text { sur les truies } \\
\text { non gravides } \\
(\%)\end{array}$} \\
\hline & & total & gravides & $19-25 \mathrm{j}$ & après $25 \mathrm{j}$ & & \\
\hline A & $\begin{array}{l}\text { Témnoin } \\
\text { I }\end{array}$ & $\begin{array}{l}1 \geq 1 \\
1 \geq 4\end{array}$ & $\begin{array}{l}98 \\
94\end{array}$ & $\begin{array}{l}5 \\
7\end{array}$ & $\begin{array}{l}18 \\
23\end{array}$ & $\begin{array}{l}85,1 \\
81,4\end{array}$ & $\begin{array}{l}21,7 \\
23,3\end{array}$ \\
\hline$B$ & $\begin{array}{c}\text { Témoin } \\
\text { I }\end{array}$ & $\begin{array}{l}116 \\
116\end{array}$ & $\begin{array}{r}99 \\
106 i\end{array}$ & $\begin{array}{l}7 \\
0\end{array}$ & $\begin{array}{l}10 \\
10\end{array}$ & $\begin{array}{l}91, \mathbf{1} \\
91, \mathbf{1}\end{array}$ & $\begin{array}{r}41,2 \\
0,0\end{array}$ \\
\hline $\mathrm{C}$ & $\begin{array}{c}\text { Témoin } \\
I\end{array}$ & $\begin{array}{l}51 \\
57\end{array}$ & $\begin{array}{l}42 \\
48\end{array}$ & $\begin{array}{l}3 \\
3\end{array}$ & $\begin{array}{l}6 \\
6\end{array}$ & $\begin{array}{l}88,2 \\
89,4\end{array}$ & $\begin{array}{l}33,3 \\
33,3\end{array}$ \\
\hline$A+B+C$ & $\begin{array}{c}\text { Témoin } \\
\text { I }\end{array}$ & $\begin{array}{l}288 \\
297\end{array}$ & $\begin{array}{l}239 \\
248\end{array}$ & $\begin{array}{l}15 \\
10\end{array}$ & $\begin{array}{l}34 \\
39\end{array}$ & $\begin{array}{l}88,1 \\
86,8\end{array}$ & $\begin{array}{l}30,6 \\
20,4\end{array}$ \\
\hline $\mathrm{D}$ & $\begin{array}{c}\text { Témoin } \\
\text { II }\end{array}$ & $\begin{array}{l}39 \\
43\end{array}$ & $\begin{array}{l}29 \\
33\end{array}$ & $\begin{array}{l}1 \\
9\end{array}$ & $\begin{array}{l}9 \\
1\end{array}$ & $\begin{array}{l}76,9^{*} \\
97,7^{*}\end{array}$ & $\begin{array}{l}10,0 \\
90,0\end{array}$ \\
\hline & $\begin{array}{l}\text { Témoin } \\
\text { II }\end{array}$ & $\begin{array}{l}79 \\
74\end{array}$ & $\begin{array}{l}55 \\
51\end{array}$ & 2 & $\begin{array}{l}22 \\
23\end{array}$ & $\begin{array}{l}72,1 \\
68,9\end{array}$ & $\begin{array}{l}8,3 \\
0,0\end{array}$ \\
\hline $\mathrm{D}+\mathrm{E}$ & $\begin{array}{l}\text { Témoin } \\
\text { II }\end{array}$ & $\begin{array}{l}118 \\
117\end{array}$ & $\begin{array}{l}84 \\
84\end{array}$ & $\begin{array}{l}3 \\
9\end{array}$ & $\begin{array}{l}31 \\
24\end{array}$ & $\begin{array}{l}73,7 \\
79,4\end{array}$ & $\begin{array}{r}8,8 \\
27,3\end{array}$ \\
\hline
\end{tabular}

Produit I : 2 mg de valérate d'œstradiol, $5 \mathrm{mg}$ de propionate de testostérone/truie.

Produit II : $2 \mathrm{mg}$ de valérianate d'œestradiol, $5 \mathrm{mg}$ d'œnanthate de testostérone/truie.

$* \mathrm{P}<0,01$.

Exactitude : (nombre de diagnostics exacts/nombre de diagnostics) $\times 100$.

N. B. : Pour tous les lots, sauf un, l'exactitude sur les truies gravides est de 100 p. 100 ; pour l'élevage E, produit II, exactitude : 99 p. 100.

Dans les trois élevages où a été utilisé le mélange valérate d'œstradiol et propionate de testostérone (produit I), l'exactitude totale est de l'ordre de 87 p. roo ; sur les truies non gravides, elle n'est que de $25 \mathrm{p}$. I 00 environ. Dans les deux cas, elle est indépendante du produit administré $(P<0,05)$. Dans les deux élevages où a été utilisé le mélange valérianate d'œstradiol et l'œnan- 
thate de testostérone (produit II), l'exactitude totale est plus faible car elle n'est que de 75 p. Ioo. Si, globalement, l'analyse ne permet pas de mettre en évidence une différence significative entre témoins et traitées, il faut signaler pour l'élevage $\mathrm{D}$, une différence nette $(\mathrm{P}<\mathrm{o}, \mathrm{OI})$ entre ces deux groupes d'animaux. Dans cet élevage, les truies sont attachées; elles sont amenées dans la case du verrat lorsqu'il y a un doute sur leur comportement.

Les résultats obtenus par administration du mélange hormonal ou du produit témoin 25 jours après l'insémination sont dans le tableau 3. L'exactitude totale est de l'ordre de 90 p. Ioo, alors qu'elle n'est que de $25 \mathrm{p}$. Ioo environ sur les animaux non gravides; dans les deux cas, elle est indépendante de la nature du produit reçu $(\mathrm{P}<0,05)$.

\section{TABLEAU 3}

Diagnostic de gestation par induction du comportement d'estrus 25 jours après insémination chez la Truie

\begin{tabular}{|c|c|c|c|c|c|c|c|}
\hline \multirow{2}{*}{ Élevage } & \multirow{2}{*}{ Produit } & \multicolumn{2}{|c|}{ Nombre de truies } & \multicolumn{2}{|c|}{$\begin{array}{c}\text { Truies non gravides } \\
\text { cestrus } \\
\text { après insémination }\end{array}$} & \multirow{2}{*}{$\begin{array}{l}\text { Exactitude } \\
\text { totale } \\
(\%)\end{array}$} & \multirow{2}{*}{$\begin{array}{c}\text { Exactitude } \\
\text { sur les truies } \\
\text { non gravides } \\
(\%)\end{array}$} \\
\hline & & total & gravides & $25-31 j$ & après $31 \mathrm{j}$ & & \\
\hline$G$ & $\begin{array}{l}\text { Témoin } \\
\text { I }\end{array}$ & $\begin{array}{l}65 \\
68\end{array}$ & $\begin{array}{l}56 \\
65\end{array}$ & $\begin{array}{l}1 \\
2\end{array}$ & $\begin{array}{l}8 \\
1\end{array}$ & $\begin{array}{l}87,7 \\
98,5\end{array}$ & $\begin{array}{l}11,1 \\
66,6\end{array}$ \\
\hline $\mathrm{H}$ & $\begin{array}{c}\text { Témoin } \\
\text { II }\end{array}$ & $\begin{array}{l}52 \\
52\end{array}$ & $\begin{array}{l}41 \\
42\end{array}$ & $\begin{array}{l}3 \\
3\end{array}$ & $\begin{array}{l}8 \\
7\end{array}$ & $\begin{array}{l}84,6 \\
86,5\end{array}$ & $\begin{array}{l}27,3 \\
30,0\end{array}$ \\
\hline I & $\begin{array}{c}\text { Témoin } \\
\text { II }\end{array}$ & $\begin{array}{l}29 \\
27\end{array}$ & $\begin{array}{l}27 \\
25\end{array}$ & $\begin{array}{l}0 \\
0\end{array}$ & $\begin{array}{l}2 \\
2\end{array}$ & $\begin{array}{l}93,1 \\
92,6\end{array}$ & $\begin{array}{l}0,0 \\
0,0\end{array}$ \\
\hline $\mathrm{G}+\mathrm{H}+\mathrm{I}$ & $\begin{array}{l}\text { Témoin } \\
\mathbf{I}+\mathrm{II}\end{array}$ & $\begin{array}{l}146 \\
147\end{array}$ & $\begin{array}{l}124 \\
132\end{array}$ & $\begin{array}{l}4 \\
5\end{array}$ & $\begin{array}{l}18 \\
10\end{array}$ & $\begin{array}{l}87,7 \\
93,2\end{array}$ & $\begin{array}{l}18,2 \\
33,3\end{array}$ \\
\hline
\end{tabular}

Produit I : $2 \mathrm{mg}$ de valérate d'œstradiol, $5 \mathrm{mg}$ de propionate de testostérone/truie.

Produit II : $2 \mathrm{mg}$ de valérianate d'œstradiol, $5 \mathrm{mg}$ d'œnanthate de testostérone/truie.

Exactitude : (nombre de diagnostics exacts/nombre de diagnostics) $\times 100$.

N. B. : l'exactitude sur les truies gravides est 100 p. 100 dans chaque lot.

TABLEAU 4

Fertilité des truies inséminées lors du retour en oestrus

\begin{tabular}{|c|c|c|c|c|}
\hline \multirow{2}{*}{ Lot } & & \multicolumn{3}{|c|}{ Moment du retour en œstrus } \\
\hline & & entre $19-25$ jours & après 25 jours & total \\
\hline $\begin{array}{l}\text { Produit } \\
\text { Témoin }\end{array}$ & $\begin{array}{l}\text { Truies réinséminées } \\
\text { Truies gravides }\end{array}$ & $\begin{array}{l}41 \\
21(51,2 \%)\end{array}$ & $\begin{array}{l}81 \\
22(27,2 \%)\end{array}$ & $\begin{array}{r}122 \\
43(35,2 \%)\end{array}$ \\
\hline $\begin{array}{l}\text { Produit } \\
\text { I + II }\end{array}$ & $\begin{array}{l}\text { Truies réinséminées } \\
\text { Truies gravides }\end{array}$ & $\begin{array}{l}38 \\
16(42,1 \%)\end{array}$ & $\begin{array}{l}71 \\
21(29,6 \%)\end{array}$ & $\begin{array}{l}109 \\
37(33,9 \%)\end{array}$ \\
\hline
\end{tabular}

$(\%)$ : fertilité. 
Au cours de cette deuxième expérience, le taux des retours en chaleurs 25 jours au moins après insémination est égal à $\mathrm{I} 5 \mathrm{p}$. Ioo environ; il ne dépend pas de la nature du produit utilisé quel que soit le moment de son administration.

Par ailleurs, la fertilité des truies réinséminées lors du retour en chaleurs pendant la période I9-25 jours semble plus faible pour les animaux qui ont reçu les stéroïdes (produits I ou II) par rapport à ceux qui n'ont eu que le solvant (tabl. 4).

Les résultats obtenus dans la troisième expérience par la technique de la biopsie vaginale sont présentés dans le tableau 5. L'exactitude totale est égale à 9ı,6 p. ıoo, elle varie avec le moment auquel est prélevé la biopsie : elle est supérieure à 90 p. Ioo lorsque l'intervalle insémination-prélèvement est supérieur à $2 \mathrm{I}$ jours, elle est inférieure à ce taux dans le cas contraire.

TABLEAU 5

Diagnostic de gestation par biopsie vaginale chez la Truie

\begin{tabular}{|c|c|c|c|c|c|c|c|c|}
\hline \multirow{2}{*}{\multicolumn{2}{|c|}{ Biopsies vaginales }} & \multicolumn{7}{|c|}{ Moment du prélèvement (jours après insémination) } \\
\hline & & $18-19$ & $20-21$ & $22-23$ & $24-25$ & $26-27$ & $28-29$ & total \\
\hline $\begin{array}{r}\text { Truies gravides } \ldots \ldots \ldots \\
\text { Exactitude } \ldots \ldots\end{array}$ & $\begin{array}{c}n \\
(\%)\end{array}$ & $\begin{array}{c}5 \\
(80,0)\end{array}$ & $\begin{array}{c}47 \\
(97,8)\end{array}$ & $\begin{array}{c}133 \\
(96,2)\end{array}$ & $\begin{array}{c}216 \\
(97,2)\end{array}$ & $\begin{array}{c}121 \\
(97,5)\end{array}$ & $\begin{array}{c}51 \\
(9 / 4,1)\end{array}$ & $\begin{array}{c}573 \\
(96,7)\end{array}$ \\
\hline $\begin{array}{r}\text { Truies non gravides } \ldots . \\
\text { Exactitude } \ldots .\end{array}$ & $\begin{array}{c}n \\
(\%)\end{array}$ & $\begin{array}{c}8 \\
(62,5)\end{array}$ & $\begin{array}{c}13 \\
(46,1)\end{array}$ & $\begin{array}{c}29 \\
(72,4)\end{array}$ & $\begin{array}{c}53 \\
(71,7)\end{array}$ & $\begin{array}{c}28 \\
(78,6)\end{array}$ & $\begin{array}{c}13 \\
(84,6)\end{array}$ & $\begin{array}{c}144 \\
(71,5)\end{array}$ \\
\hline $\begin{array}{l}\text { avec un retour } \\
\text { normal }\left(^{1}\right) \ldots \ldots \ldots \\
\text { Exactitude } \ldots \ldots\end{array}$ & $\begin{array}{c}n \\
(\%)\end{array}$ & $\begin{array}{c}6 \\
(83,3)\end{array}$ & $\begin{array}{c}7 \\
(85,7)\end{array}$ & $\begin{array}{c}9 \\
(77,7)\end{array}$ & $\begin{array}{r}6 \\
(100,0)\end{array}$ & $\begin{array}{c}2 \\
(50,0)\end{array}$ & $\begin{array}{c}2 \\
(50,0)\end{array}$ & $\begin{array}{c}32 \\
(81,2)\end{array}$ \\
\hline $\begin{array}{l}\text { avec un retour } \\
\text { anormal }\left({ }^{1}\right) \ldots \ldots \\
\text { Exactitude } \ldots \ldots\end{array}$ & $\begin{array}{c}n \\
(\%)\end{array}$ & $\begin{array}{c}2 \\
(0,0)\end{array}$ & $\begin{array}{c}6 \\
(0,0)\end{array}$ & $\begin{array}{c}20 \\
(70,0)\end{array}$ & $\begin{array}{c}47 \\
(68,0)\end{array}$ & $\begin{array}{c}26 \\
(80,7)\end{array}$ & $\begin{array}{c}11 \\
(90,9)\end{array}$ & $\begin{array}{c}112 \\
(68,7)\end{array}$ \\
\hline Exactitude totale & $(\%)$ & $(69,2)$ & $(86,6)$ & $(92,0)$ & $(91,8)$ & $(94,0)$ & $(92,2)$ & $(91,6)$ \\
\hline
\end{tabular}

(1) Retour en ostrus normal 18-25 jours après insémination. Retour anormal $>25$ jours.

Exactitude : (nombre de diagnostics exacts/nombre de diagnostics) $\times 100$.

TABLEAU 6

Taux d'utilisation des biopsies vaginales chez la Truie

Nombre total $782(100)$. Nombre de diagnostics $717(91,7)$

$\left.\begin{array}{c|c|l}33(4,2) & \text { Réforme sans aucun contrôle } \\ 8(1,0) & \text { Erreur d'identification } \\ 10(1,2) & \text { Erreur technique } \\ 1{ }^{\prime}(1,8) & \text { Mauvaise biopsie (sans épithélium) }\end{array}\right\}(4,0)$

Par cette technique, l'exactitude obtenue sur les truies gravides est égale à 96,7 p. Ioo, elle n'est que de 7 r,5 p. Ioo sur les animaux non gravides. Cependant, 8I,2 p. I oo des truies qui reviennent en chaleurs entre le $18^{\mathrm{e}}$ et le $25^{\mathrm{e}}$ jour après saillie sont diagnostiquées non gravides et 68,7 p. Ioo pour celles qui reviennent après le $25^{\mathbf{e}}$ jour. 
Il faut signaler que 9I,7 p. Ioo des biopsies prélevées seulement ont fait l'objet d'un diagnostic. Les raisons d'élimination des prélèvements sont énumérées dans le tableau 6 ; on peut remarquer que la moitié des biopsies non exploitées (soit + p. Ioo du total) est imputable à la technique.

\section{DISCUSSION}

Les résultats des deux premières expériences indiquent qu'une bonne observation des retours en cestrus est apparemment une technique convenable de diagnostic de gestation. F,n effet, l'exactitude totale ou l'exactitude sur les animaux gravides qui constituent la majorité des truies après saillie sont élevées ce qui a déjà été signalé (JöCHLE et SCHILLING, I965; JöchlE et al., I967 ; ROTHE, I970 ; BECzE et SzILvASSY, I97I ; ANDR̈̈, I969; MAIRE et al, I973).

Cependant, le critère de diagnostic s'applique essentiellement aux truies non gravides et les résultats montrent que l'exactitude obtenue sur ces dernières est faible. Il faut souligner à ce sujet la variabilité constatée entre les élevages. Par contre, lorsque la détection des chaleurs est accomplie avec le verrat, l'exactitude sur les animaux qui ne mettent pas bas augmente comme l'indiquent les résultats de la première expérience et ceux de l'élevage $D$ au cours de la deuxième expérience.

Les résultats montrent aussi que 1'utilisation d'un mélange œstrogène-androgène n'améliore pas significativement la précision du diagnostic par rapport à celle qui est observée avec un produit témoin. Cette constatation a été déjà relevée lorsque les animaux traités ont été comparés à un lot de référence (JöchrE et SchrLLING, I965). Pourtant la dose du composé œstrogénique utilisé est largement supérieure à celle qui provoque des chaleurs de durée normale chez la Truie castrée et à celle audessus de laquelle celles-ci viennent toutes en ostrus (Signoret, I967).

Compte tenu du rôle de la progestérone sur l'apparition du comportement d'ostrus (SIGNORET, I97I) et de l'évolution du taux de cette hormone chez la Truie non gravide par défaut de fécondation ou par mortalité embryonnaire précoce, il est normal que l'œestrus doive être induit pendant la période à laquelle il devrait se manifester. Un traitement réalisé entre le $16^{\circ}$ et le $22^{\circ}$ jour après $1^{\prime}$ insémination correspond au moment optimum compte tenu de la durée moyenne du cycle ostrien et de sa variabilité (SIgNORET, I967). Il semble alors logique d'observer les chaleurs éventuellement induites dans les 4 jours qui suivent le traitement ce qui n'est pas toujours défini de cette façon. Les résultats obtenus par un traitement 25 jours après saillie confirment ce fait. L'exactitude totale est élevée mais cela peut être attribué à l'importance des truies gravides par rapport à l'ensemble des animaux soumis au diagnostic à ce moment. Èn effet une grande partie des truies non gravides sont déjà revenues en chaleurs et ne sont pas traitées de ce fait.

Enfin la fertilité des animaux réinséminés lors de l'œestrus induit semble plus faible que celle des animaux témoins saillis dans les mêmes conditions ; cette baisse de fertilité a déjà été observée avec 1'utilisation de ce complexe hormonal comme diagnostic de gestation (ANDRÄ et al., I969; RoTHE, I970; BECzE et SzILVASSY, I97I) ou comme moyen de grouper les chaleurs au sevrage (BIELANSKI et WIERzchos, I974; Smidt et al., I968). De plus il faut souligner la faible fertilité des truies réinséminées lors d'un retour en chaleurs tardif (a près 25 jours), car elle est inférieure à 30,0 p. IOO. 
Quant à la technique de la biopsie vaginale, on peut penser qu'elle est moins adaptée aux conduites d'élevage habituelles. Un des problèmes soulevés par 1'utilisation d'une technique systématique est celui du nombre de prélèvements ininterprétables comme MAIRE, MAURY et Poline (I973) l'ont souligné. Ces auteurs ont été amenés à rejeter I 6 p. Ioo de leurs échantillons. Dans l'étude qui vient d'être rapportée, ce taux n'est que de $4 \mathrm{p}$. Ioo. Les résultats obtenus confirment d'ailleurs la plupart de ceux qui ont été publiés à ce sujet (GIRARDOT, I97I ; WALKER, I972 ; Rommer et al., I973; Smidt, I973). Contrairement à la technique de l'induction de l'œstrus, l'exactitude obtenue sur les truies gravides est légèrement inférieure à roo p. roo. Par contre l'exactitude sur les truies non gravides est plus élevée et on peut déceler précocement dans $70 \mathrm{p}$. Ioo des cas environ 1'absence de gestation pour les truies qui manifestent tardivement des chaleurs. On peut donc penser qu'une part non négligeable des anœestrus sans gestation correspondent à des ovulations silencieuses ou à une mauvaise détection de l'œstrus. Pour les autres cas d'anœstrus qui ne peuvent pas être distingués des animaux gravides, on peut supposer qu'il y a maintien des corps jaunes ce qui a été constaté par ROBERTSON et SARDA (I973) par dosage de la progestérone plasmatique. Ces cas rendent peut-être compte d'une mortalité embryonnaire importante et d'une occupation insuffisante de l'utérus. Dans ces conditions en effet le retour en chaleurs est différé de plusieurs jours (PoLGE, Rowson et Chang, Ig66).

Une bonne détection de l'œstrus peut constituer une technique de diagnostic de gestation satisfaisante car la précision obtenue sur les animaux gravides est égale à ıoo p. Ioo.

Le taux d'exactitude sur les truies non gravides dépend surtout de la qualité de la détection de l'œestrus plutôt que de l'emploi d'un procédé hormonal. Ce dernier n'élimine pas la surveillance à effectuer ce que permet par contre l'utilisation systématique d'une technique comme la biopsie vaginale.

Reçu pour publication en mai 1975.

\section{REMERCIEMENTS}

Cette étude a été réalisée grâce à la collaboration active des personnes suivantes: MM. Albert, Breillad Frères, Barranger, Bernardin, Cartron, Couteleau, Cornu, Dutertre, Hervouet, Gautier, Gruand, Leclerc, Ieggentilhomme, Mottard, Naveau, Pouget, Rallu, Renoux, Reybaud, Saulnier, Talbot, Vexiau;

- d'organismes professionnels : E. D. E. de la Mayenne, E. D. E. des Deux-Sèvres, Syndicat d'Élevage Porcin de la Vendée :

- de groupements de producteurs : C. A. R. C. O. (Deux-Sèvres), G. A. P. P. E. V. (Vendée), G. E. C. O. (Deux-Sèvres), G. E. R. C. O. (Deux-Sèvres) S. O. C. A. V. E. M. (Mayenne)

Nous tenons à leur présenter nos plus vifs remerciements. Nous remercions aussi MM. OLLIvier, Legault, responsables du Centre Expérimental de Sélection Porcine de l'I. N. R. A,, le Docteur Freudenberg Veb Jenapharm (R. D. A.)

Ce travail a pu être effectué à l'aide d'un financement du F. O. R. M. A. au titre d'une convention passée entre cet organisme, l'I. T. P. et l'I. N. R. A. pour la réalisation d'un programme de Rationalisation de la Production Porcine. 


\author{
SUMMARY
}

\author{
STUDY OF TWO TECHNIQUES FOR THE DIAGNOSIS OF PREGNANCY IN THE SOW
}

Two techniques for the diagnosis of pregnancy in the sow have been studied.

The first one is based on recording of an eventual return to oestrus i 8 to 25 days after the reference insemination. Total accuracy varied according to herds from 73 to $93 \mathrm{p}$. Ioo. It represented 100.o p. Ioo for pregnant sows and was very low for non-pregnant sows. In the latter, the accuracy was not improved by injection of an oestrogen-androgen mixture 19 days after the insemination. Neither was it improved when the treatment was applied 25 days after mating and when oestrus was detected between day 25 and $3 \mathrm{r}$.

The second technique was based on vaginal biopsy. Total accuracy was around $92 \mathrm{p}$. Ioo. It represented $96.7 \mathrm{p}$. Ioo for the pregnant sows and $71.5 \mathrm{p}$. Ioo for the non-pregnant ones. Using this technique, the absence of pregnancy can be detected in $68 \mathrm{p}$. 100 of the animals exhibiting late returns to oestrus.

\title{
RÉFÉRENCES BIBLIOGRAPHIQUES
}

Andrä B., Bergfeld J., Chemnitius H. K., Jähne H., Rommel P., Schmidt W., Stahl U., 1969. "Gravignost Schweine " ein neues Präparat für die TrächtigKeitxfeststallung beim Schwein. Fortpfl Bes. Aufz. Haust., 5, 327-334.

Becze J., Szilvássy B., I97r. Early diagnosis of pregnancy in pigs starting the cestrus following parturition and the treatment of anaoestrus with. Gravignost. Magy. Allatorv Lap., 26, 568-570.

Bielax́ski A., Wierzchoś E., I974. Próba wywotania synchronizowanej owulacji u loch przy użycin preparatu. Gravignost. Medycyna Weterynaryjna, 30, I75-I76.

Caugant A., 1974. Combien coûtent nos mauvaises techniques. Bulletin I. T. P., 4, 19-24.

Girardot J., r97. Diagnostic de gestation chez la Truie. Mémoive de Fin d'Études de l'E. N.S. A. de Nancy. Station de Physiologie de la Reproduction. I. N. R. A., 37380 Nouzilly.

Girardot J., Bosc M. J., Bariteau F., 1972. Diagnostic de gestation par Biopsie vaginale chez la Truie. Journées de la Recherche Porcine en France, 59-62, I. N. R. A., I. T. P. éd., Paris.

Jöchle W., Schilling E., I965. Improvement of conception rate and diagnosis of pregnancy in sows by an androgen-oestrogen depot preparation. J. Reprod. Fertil., 10, 439-440.

Jöchle W., Schilling F., Smidt D., Fiedler E., I967. Effets d'une association androgène-cestrogène sur les fonctions de reproduction chez la Truie Zuchthygiene, 2, I22-I27.

Maire C., Maury Y., Poline F., i973. Détermination précise du moment de l'cstrus et confirmation de l'état de gravidité. Journées de la Recherche Porcine en France, 33-37, I. N. R. A., I. T. P. éd. Paris.

Polge C., Rowson I. E. A., Change M. C., I966. The effect of reducing the number of embryos during early stages of gestation on the maintenance of pregnancy in the pig. J. Reprod. Fert., 12 , 395-397.

Robertson H. A., SARda I. R., r97I. A very early pregnancy test for mammals : its application to the cow, ewe and sow. J. Endocr., 49, 407-419.

Robertson H. A., King G. J., I974. Plasma concentrations of progesterone, osstrone, oestradiol-I $7 \beta$ and of oestrone sulphate in the pig at implantation, during pregnancy and at parturition. J. Reprod. Fert., 40, I33-I4I.

Rommel P., HüHN U., Kahler G., I973. FrühträchtigKeitsnach weis beim Schwein. I. Mit vaginalhistologische Methode. Monatshefte für Veterinärmedizin, 28, 458-462.

Rothe K., I97o. Die Hormonale Trächtigketisdiagnose beim schwein. Cong. de Wels (Autriche). Dtsch. Eieväratl. Wschr., 78, 73-148.

Signoret J. P., 1967. Durée du cycle et de l'œstrus chez la Truie. Action du benzoate d'œestradiol chez la femelle ovariectomisée. Ann. Biol. anim. Bioch. Biophys., $7,407-42 \mathrm{r}$.

Signoret J. P., I97I. Étude de l'action inhibitrice de la progestérone sur l'apparition du comportement sexuel induit par injection d'œstrogènes chez la Truie et la Brebis ovariectomisées. Ann. Biol. anim. Bioch. Biophys., 11, $489-494$.

Signoret J. P., Du Mesnil du Buisson F., i96r. Étude du comportement de la Truie en wstrus. IVe Cong. intern. Reprod. anim., La Haye, I I-I75. 
Shearler I. J., Purvis K., Jenkin G., Haynes N. B., I972. Peripheral plasma progesterone and oestradiol-i $\beta \beta$ levels before and after puberty in gilts. J. Reprod. Fert., 30, 347-360.

Smidt D., Jöснle W., Thume O., r968. Heat synchronization by Androgen Estrogen Combinations in sows and Gilts. VI Cong. Intern. Reprod. Anim. Insem. Artif., Paris, II, I523-I526.

Sмгdт D., 1973. TrächtigKeitsdiagnose beim schwein unter BerücKsichtigung praxisnaher Methoden. In Aktuelle Themen der Tierernährung und Veredlungswirtschaft, Wiss. Tag. Lohmann, Cuxchaven (Novembre 1972).

Stabenfeldt G. H., Akins E. L., Ewing $\quad$ L. L., Morrissftte $\quad$ M. C., Ig69. Peripheral plasma progesterone levels in pigs during the oestrous cycle. $J$. Reprod. Fert., 20, 443-449.

Walker D., I972. Pregnancy I iagnosis in Pigs. Vet. Rec., 90, I39-I 44. 